\title{
Gpr17 deficiency in POMC neurons ameliorates the metabolic derangements caused by long-term high-fat diet feeding
}

\author{
Austin M. Reilly ${ }^{1}$, Shudi Zhou', Sunil K. Panigrahi², Shijun Yan ${ }^{3,4}$, Jason M. Conley ${ }^{3,4}$, Patrick L. Sheets ${ }^{1,5}$,
} Sharon L. Wardlaw ${ }^{2}$ and Hongxia Ren (1) 1,3,4,5,6,7

\begin{abstract}
Background: Proopiomelanocortin (POMC) neurons in the arcuate nucleus of the hypothalamus (ARH) control energy homeostasis by sensing hormonal and nutrient cues and activating secondary melanocortin sensing neurons. We identified the expression of a G protein-coupled receptor, Gpr17, in the ARH and hypothesized that it contributes to the regulatory function of POMC neurons on metabolism.

Methods: In order to test this hypothesis, we generated POMC neuron-specific Gpr17 knockout (PGKO) mice and determined their energy and glucose metabolic phenotypes on normal chow diet (NCD) and high-fat diet (HFD).

Results: Adult PGKO mice on NCD displayed comparable body composition and metabolic features measured by indirect calorimetry. By contrast, PGKO mice on HFD demonstrated a sexually dimorphic phenotype with female PGKO mice displaying better metabolic homeostasis. Notably, female PGKO mice gained significantly less body weight and adiposity $(p<0.01)$, which was associated with increased energy expenditure, locomotor activity, and respiratory quotient, while males did not have an overt change in energy homeostasis. Though PGKO mice of both sexes had comparable glucose and insulin tolerance, detailed analyses of liver gene expression and serum metabolites indicate that PGKO mice could have reduced gluconeogenesis and increased lipid utilization on HFD. To elucidate the central-based mechanism(s) underlying the better-preserved energy and glucose homeostasis in PGKO mice on HFD, we examined the electrophysiological properties of POMC neurons and found Gpr17 deficiency led to increased spontaneous action potentials. Moreover, PGKO mice, especially female knockouts, had increased POMC-derived alpha-melanocyte stimulating hormone and beta-endorphin despite a comparable level of prohormone POMC in their hypothalamic extracts.
\end{abstract}

Conclusions: Gpr17 deficiency in POMC neurons protects metabolic homeostasis in a sex-dependent manner during dietary and aging challenges, suggesting that Gpr17 could be an effective anti-obesity target in specific populations with poor metabolic control.

Correspondence: Hongxia Ren (renh@iu.edu)

${ }^{1}$ Stark Neurosciences Research Institute, Medical Neuroscience Graduate

Program, Indiana University School of Medicine, 320 W. 15th Street Indianapolis, Indianapolis, IN 46202, USA

${ }^{2}$ Department of Medicine, Division of Endocrinology, Vagelos College of Physicians and Surgeons, Columbia University, New York, NY 10032, USA

Full list of author information is available at the end of the article.

\section{Introduction}

Chronic food intake in excess of energy expenditure leads to adiposity gain and obesity. Proopiomelanocortin (POMC) neurons in the arcuate nucleus of the hypothalamus (ARH) control energy homeostasis through the production of alpha-melanocyte stimulating hormone $(\alpha-$ MSH), a POMC-derived peptide which activates melanocortin receptors 3 and $4(\mathrm{MC} 3 / 4 \mathrm{R})^{1-5}$. Human patients 
with loss-of-function mutations in $P O M C$ or $M C 3 / 4 R$ have persistent hunger and consequently develop obesity ${ }^{5,6}$, highlighting the crucial role of melanocortin signaling in managing energy balance. Neurons coexpressing Agouti-related peptide and Neuropeptide $\mathrm{Y}$ (AgRP/NPY) in the ARH decrease satiety by opposing the functions of POMC neurons via GABAergic projections onto POMC neurons and the secretion of AgRP and NPY neuropeptides $^{7-12}$. POMC neurons and AgRP/NPY neurons have divergent responses to adiposity signals ${ }^{13-15}$. Given the pivotal role of POMC neurons in regulating metabolic homeostasis, our research objective was to identify novel mechanisms controlling POMC neuronal activity that can be leveraged for treating obesity.

Although the role of POMC neurons in managing energy balance is well established, the biological mechanisms regulating their activity is still an area under active investigation. Forkhead box protein O1 (FoxO1) protein was detected in the hypothalamic AgRP and POMC neurons, and hypothalamic expression of a constitutive active form of FoxO1 resulted in a loss of the ability of leptin to curtail food intake ${ }^{16}$. Carboxypeptidase $\mathrm{E}$ (Cpe), an enzyme that mediates POMC processing, was identified as a FoxO1 transcriptional target in POMC neurons ${ }^{17}$. We, as well as other groups, identified Gpr17 as a transcriptional target of FoxO1 in the central nervous system $^{18,19}$. Furthermore, we generated Gpr17 conditional knockout mice and analyzed its metabolic function in AgRP neurons ${ }^{18}$.

Based on emerging evidence that the orphan receptor Gpr17 is expressed by neuronal populations involved in energy homeostasis ${ }^{18,20}$, we hypothesized that Gpr17 signaling regulates POMC neuronal function to control appetite, metabolism, and energy homeostasis. In order to test this hypothesis, we generated POMC neuronspecific Gpr17 knockout mice and determined their basal metabolic features. Gender differences exist in regulation of metabolism $^{21}$. POMC neurons exhibit sexual dimorphism in the regulation of energy homeostasis ${ }^{22,23}$. Moreover, aging and unhealthy diet are known factors associated with adiposity gain, a major contributor to insulin resistance and metabolic derangements. Therefore, in this study, we analyzed the metabolic phenotype of both female and male mice at different ages challenged with chronic feeding of high-fat diet. Our systemic characterization of the mutant mice of both sexes revealed that Gpr17 deficiency in POMC neurons ameliorated the metabolic derangements caused by long-term high-fat diet feeding, which was more pronounced in female mice.

\section{Materials and methods \\ Experimental animals}

Pomc promoter-driven Gpr17 knockout (PGKO) mice were generated by cross-breeding Pomc-Cre mice ${ }^{24}$ and
Gpr17 lox/lox mice ${ }^{20}$. Pomc-Cre negative, Gpr17 lox/lox mice, or Pomc-Cre;Gpr17 lox/+mice were used as controls for all experiments. All mice were maintained in the Columbia University or IUSM Lab animal resource center (LARC) facility. The procedures were approved by the Columbia University Animal Care and Utilization Committee and Indiana University Animal Care and Use Committee (IACUC). Mice were weaned at 3 weeks old and group housed (3-5 mice per cage) except during indirect calorimetry measurements (1 mouse per cage). Animals were assigned for experiments blinded and genetic information was revealed only after experiment was completed.

\section{Feeding regimen}

Experimental animals were fed with either normal chow diet (NCD) or high-fat diet (HFD). NCD had $62.1 \%$ of calories from carbohydrates, $24.6 \%$ from protein, and 13.2\% from fat (PicoLab Rodent Diet 20, catalog \#5053; Purina Mills. In order to induce obesity, we used high-fat diet (HFD) containing 60\% calories from fat, 20\% from protein, 20\% from carbohydrate (Research Diets, catalog \#D12492, New Brunswick, NJ). We profiled the metabolic phenotype in adult cohorts fed NCD, HFD for 4-5 months and for $8-10$ months (i.e., long-term HFD) as indicated by the figure legends.

\section{Serum biochemistries}

Mouse serum was collected by tail vein bleeding or terminal cardiac puncture. In order to characterize the metabolic flexibility of mice, we analyzed serum metabolite concentrations during ad libitum feeding, short fasting during daytime $(5 \mathrm{~h})$, overnight fasting $(\sim 16 \mathrm{~h})$, and refeeding ( $4-5 \mathrm{~h}$ of ad libitum feeding after overnight fasting). We measured free glycerol (Sigma cat. no. F6428), free cholesterol (Wako cat. no. 993-02501), and non-esterified free fatty acid (Wako cat. nos. 999-34691, 995-34791, 991-34891, 993-35191) by colorimetric enzymatic assays. We used ELISAs to measure serum leptin (EMD Millipore cat. no. EZML-82K), and serum insulin (EMD Millipore cat. no. EZRMI-13K). All reactions were performed according to manufacturer protocols.

\section{Indirect calorimetry}

Indirect calorimetry measurements (food intake, energy expenditure, respiratory quotient, and locomotor activity) were collected using a TSE PhenoMaster Platform (TSE Systems, Chesterfield, MO) as described previously ${ }^{25}$, except for the female long-term HFD cohort. Briefly, mice were single-housed for the duration of metabolic analysis and were allowed to acclimate for $48 \mathrm{~h}$ before analysis. Data were collected every 51 min during a normal 12-h day/night cycle. Mice had ad libitum access to normal or high-fat diet chow. Total body weight and lean mass were 
determined beforehand by MRI scan (EcoMRI-100, EcoMRI Houston, TX) for calculating indirect calorimetry measurements. Long-term HFD females were analyzed on Comprehensive Laboratory Animal Monitoring System (CLAMS, Oxymax Windows 3.0.3; Columbus Instruments). CLAMS data were collected in the same manner at 17-30 min intervals during a normal 12-h day/ night cycle.

\section{Quantification of POMC and POMC-derived peptides}

PGKO and control mice aged 3-6 months were exposed to HFD for 2 weeks, then sacrificed after ad libitum feeding and peptides were measured in acid extracts of dissected medial basal hypothalamic samples as previously described ${ }^{26}$. POMC was measured by 2 site ELISA with antibodies provided by Dr. Anne White (University of Manchester, UK); there is no crossreactivity with $\alpha-\mathrm{MSH}$ or $\beta-\mathrm{EP}^{26}$. $\alpha$-MSH and $\beta$-EP were measured by radioimmunoassay (RIA) ${ }^{26}$. The $\beta$-EP antibody fully cross-reacts with $\beta$-EP (1-31), $\beta$-EP $(1-26)$, and $\beta$-EP (1-27) and $2.6 \%$ with POMC on a molar basis. The $\alpha-\mathrm{MSH}$ antibody fully cross-reacts with desacetyl $\alpha$-MSH; there is no cross-reactivity with $\beta$-EP, adrenocorticotropin hormone $(\mathrm{ACTH})$, or POMC. The total protein concentration was measured in each hypothalamic sample and used for normalization in order to minimize the variability of individual sample dissection and extraction.

\section{Glucose tolerance test and insulin tolerance test}

Glucose tolerance test (GTT) was performed as described previously ${ }^{25}$ in PGKO and WT mice fed HFD for 4-5 months. Briefly, we injected a bolus of glucose solution intraperitoneally $(1 \mathrm{~g} / \mathrm{kg}$ in males and $2 \mathrm{~g} / \mathrm{kg}$ in females) after an overnight fast $(\sim 16 \mathrm{~h})$. Blood glucose concentrations were measured from drops of tail blood at the indicated time points using a glucose meter (Bayer Breeze2). In order to determine the sensitivity of peripheral tissues to insulin, we performed insulin tolerance tests (ITT) to mice after $6 \mathrm{~h}$ of fasting. We delivered 1.5 Units $/ \mathrm{kg}$ of insulin (Novo Nordisk) by intraperitoneal injection and recorded tail blood glucose concentration over time $(0,15,30,60,90 \mathrm{~min})$.

\section{Gene expression}

Livers and brains were collected from mice euthanized with $\mathrm{CO}_{2}$ after feeding as indicated in the corresponding figure legend. We extracted RNA with TRIzol Reagent (Invitrogen) and used Superscript II reverse transcriptase (Invitrogen) to synthesize template cDNA for quantitative RT-PCR. We PCR amplified target DNA with primers spanning introns. Primer sequences are available upon request. All reactions were performed according to manufacturer protocols.

\section{Statistical comparisons}

We used GraphPad Prism software for statistical analyses and graphics generation. Data are presented as average \pm standard error. Comparisons were made between age-matched control and knockout littermates. Statistical tests and group sizes are specified in the corresponding Figure legend. Sample exclusion criteria were predetermined. For endpoint experiments, we excluded outliers more than 2 SD from the group mean. Mice with clearly abnormal behavior, such as hyperlocomotion or lack of food intake, were excluded from metabolic analysis.

\section{Electrophysiology, histology, and fluorescence activated cell sorting (FACS)}

Detailed methods are described in 'Supplemental Methods'.

\section{Results}

PGKO male and female mice have normal body weight and composition on normal chow diet (NCD)

In order to understand the physiological role of Gpr17 in POMC neurons, we specifically knocked out Gpr17 in POMC neurons (Pomc-Cre;Gpr17 lox/lox mice, hereafter called PGKO mice). PGKO mice were compared with littermate control mice in individual cohorts (hereafter called wild-type (WT) mice).

In order to characterize the specificity and efficiency of Cre-dependent knockout, we first extracted genomic DNA from various tissues and were able to detect the recombined allele in the mediobasal hypothalamus (mbh) but not in other tissues (Fig. S1A, arrow). In order to specifically assess the gene expression of Gpr17 in POMC neurons in the WT and PGKO mice, we used the fluorescence activated cell sorting (FACS) of live dissociated hypothalamic cells. We introduced a reporter RosatdTomato to specifically label POMC neurons in WT and PGKO mice, then collected the Tomato + cell population for gene expression analysis with RT-PCR. Gpr17 transcript was virtually undetectable in the POMC neurons of the PGKO mice, while it was detected in the POMC neurons of the WT mice as well as the input fractions (Fig. S1B-D). FACS successfully enriched the Pomc transcripts ( 400 fold) in the Tomato + fraction, which further validated our method (Fig. S1E). Overall, this set of experiments demonstrated the successful and specific ablation of Gpr17 expression in the POMC neurons of PGKO mice.

We characterized body composition and energy homeostasis in PGKO mice on normal chow diet. PGKO males and females had comparable total body weight with control males and females, respectively (Fig. 1a, b). We measured body composition with MRI and found that fat mass (percentage of body weight) and lean mass 


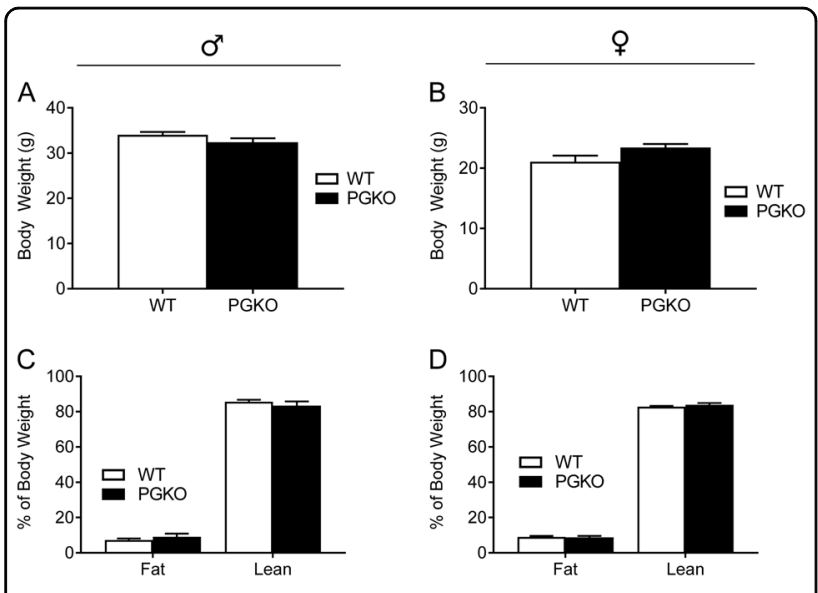

Fig. 1 PGKO male and female mice have normal body weight and composition on NCD. Left-hand data are male mice, right-hand data are female mice. Body weight in grams is presented for males (a) and females (b). Fat and lean mass percentage for male (c) and female (d) mice were measured with MRI prior to indirect calorimetry. Statistical comparisons with unpaired student's $t$-test were non-significant $(n=$ 8,7 males; $n=9,7$ females). Data shown are average \pm standard error

(percentage of body weight) were similar between PGKO and control mice (Fig. 1c, d). Therefore, we concluded that PGKO mice on a normal chow diet were able to maintain a healthy body weight and composition similarly to control mice without an apparent defect in growth or energy balance.

\section{Feeding and energy balance in NCD-fed PGKO mice}

We used indirect calorimetry to determine whether male and female PGKO mice had differences in energy homeostasis on NCD. PGKO mice showed comparable measures compared with control mice in terms of energy expenditure (i.e., heat production, Fig. 2a-d), food intake (Fig. 2e-h), respiratory quotient (Fig. 2i-l), oxygen consumption (Fig. $2 \mathrm{~m}-\mathrm{p}$ ), and locomotor activity (Fig. $2 \mathrm{q}-\mathrm{t}$ ). Since caloric intake and energy expenditure were similar between control and PGKO mice, mice did not develop different body weight/composition (Fig. 1a-d). The metabolism of glucose and fatty acids produces different respiratory quotients (volumetric $\mathrm{CO}_{2}: \mathrm{O}_{2}$ ratio) because of their different reaction stoichiometries. Respiratory quotient indicated that metabolic flexibility between carbohydrate or lipid metabolism was sustained for PGKO mice (Fig. $2 m-p$ ). Nocturnal locomotor activity (Fig. 1q-t) showed that PGKO mice had intact feeding behaviors in diurnal and nocturnal phases. Overall, PGKO mice did not have an overt metabolic phenotype when maintained on NCD.

\section{High-fat diet changes metabolism and feeding behaviors of PGKO mice in a sex-dependent manner}

Since normal chow is a nutritionally balanced diet not sufficient to cause obesity, we decided to challenge PGKO mice with high-fat diet (HFD) containing $60 \% \mathrm{kcal}$ from fat, $20 \%$ from protein, $20 \%$ from carbohydrate to reveal disparities in regulating energy homeostasis. One-year-old mice male and female mice were fed HFD for 3 and 5 months, respectively. We used indirect calorimetry to measure key metabolic features in both male and female mice and observed sexually dimorphic metabolic phenotypes. Male PGKO mice did not have statistically different energy expenditure during light or dark phases (Fig. 3a, b). By contrast, energy expenditure in female mice trended upward for light and dark phases but did not reach significance (Fig. 3c, d). Twenty-four hours of food intake was not significantly different in either the male or female groups (Fig. 3e-h). Together, these data suggested that female PGKO mice had better energy balance. Respiratory quotient was similar in male mice (Fig. $3 \mathbf{i}-\mathbf{j}$ ) but was significantly increased in female mice (Fig. 3k, l), indicating more glucose utilization in PGKO female mice. Oxygen consumption was not altered in male mice (Fig. $3 \mathrm{~m}, \mathrm{n}$ ) but was significantly increased in female mice (Fig. 3o, p). We measured locomotor activity during the light and dark phase in PGKO mice (Fig. 3q-t) and found that female mice had significantly increased locomotor activity during the dark phase. Taken together, PGKO females appeared to have better energy balance and we predicted that these mice may resist weight gain with prolonged high diet feeding.

\section{PGKO mice resisted weight and adiposity gained from long-term HFD}

In order to determine if improved energy balance predicted lower weight gain, we continued to feed HFD to the same cohorts used for the indirect calorimetry experiment (Fig. 3). Indeed, we discovered that after long-term HFD, male and female PGKO mice had lower total body weight (Fig. 4a, c, respectively) and epididymal white adipose tissue (EWAT) mass (Fig. 4b, d). Total body weight and EWAT mass trended lower in PGKO males but did not reach statistical significance $(p=0.09)$; however, weight gained from five additional months of HFD was significantly lower (Fig. 4f). The female PGKO group was even more protected from the diet-induced weight gain, having reduced body weight gain (Fig. $4 \mathrm{~g}, \mathrm{~h}$ ) and EWAT mass at the end of the experiment (Fig. $4 \mathrm{~d}$ ).

\section{PGKO mice have sex-dependent changes in serum metabolite concentrations and hepatic glycolytic enzyme expression}

In order to determine if PGKO mice had altered nutrient metabolism compared with control mice, we measured the blood glucose and the serum concentration of various metabolites in other PGKO cohorts fed HFD. We determined that blood glucose levels during fastingrefeeding, glucose tolerance, and insulin tolerance were 

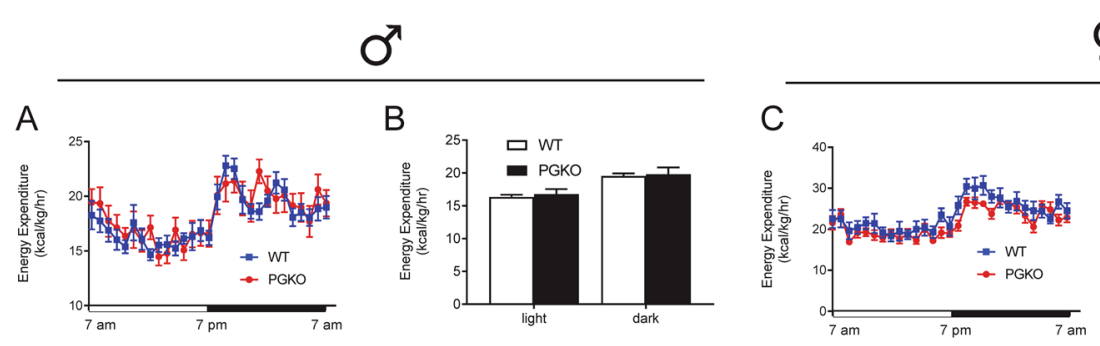

우

E

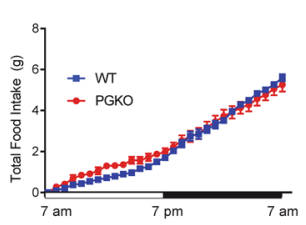

।

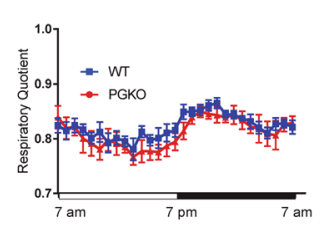

M

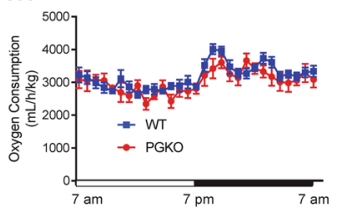

Q

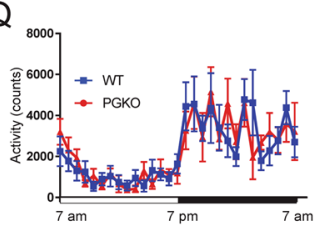

F

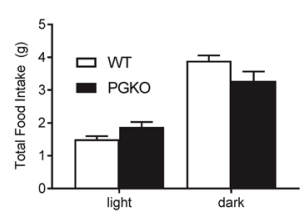

J

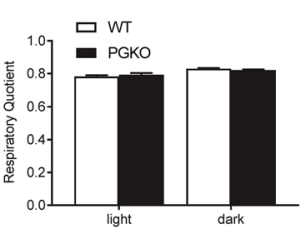

N

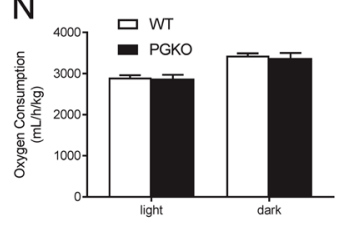

R

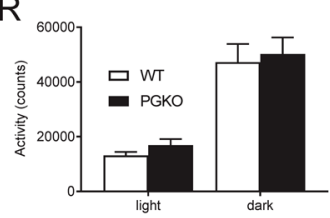

G

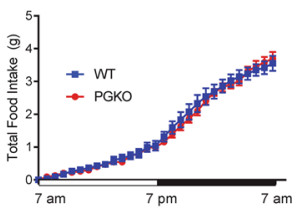

K
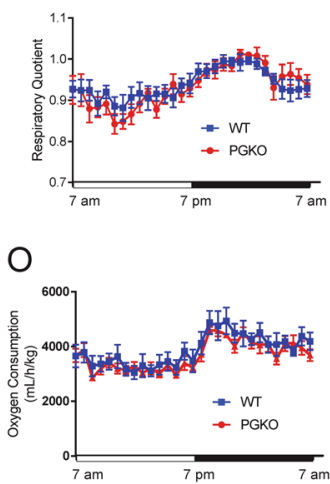

S

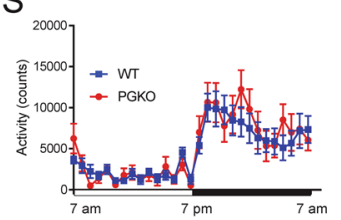

D

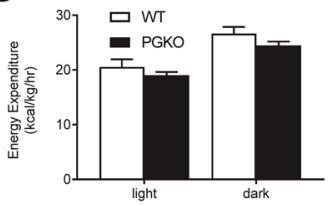

$\mathrm{H}$

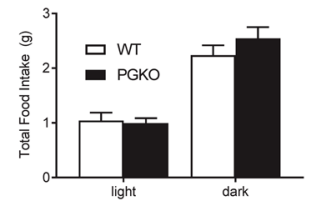

L

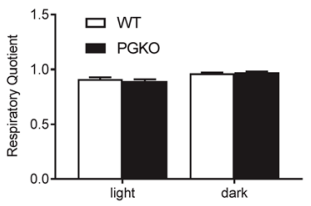

P

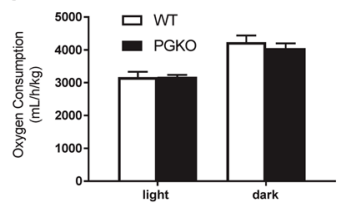

T

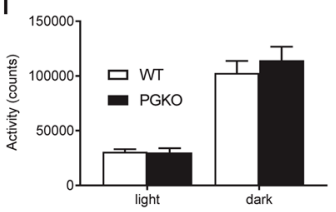

Fig. 2 PGKO mice display comparable metabolic features as wild-type mice without dietary challenge. Left-hand figures are male mice; righthand figures are female mice. We determined energy expenditure normalized to lean mass (a-d), total 24-h food intake (e-h), respiratory quotient $(\mathbf{i}-\mathbf{l})$, and oxygen consumption $(\mathbf{m}-\mathbf{p})$. Locomotor activity was measured by counting the number of floor beam breaks (q-t). Bar graphs are the 12-h average of data shown in corresponding line graph, separated into light and dark phases of the light cycle. Statistical comparisons with unpaired student's $t$-test were non-significant ( $n=8,7$ males; $n=9,7$ females). Data shown are average \pm standard error

indistinguishable in PGKO mice (Fig. S2A-F). Then, we assessed whether the expression of key hepatic genes for glucose and lipid metabolism in PGKO mice was altered. We measured hepatic mRNA transcripts involved in glycolysis, gluconeogenesis, and fatty acid synthesis for males and females. In males, we found a trending decrease in glucokinase $(G c k)$ and a significant twofold increase in pyruvate dehydrogenase kinase $4(P d k 4)$ transcript (Fig. S2G). In female PGKO mice (Fig. S2H), we found a significant increase in glucokinase expression and trend for increased Pdk4. Pck1 (encoding phosphoenylpyruvate carboxykinase) and $P d k 4$ transcripts trended higher than control mice. Glucokinase is a target gene of insulin signaling through the sterol regulatory element binding transcription factor 1 (Srebp1c), which trended lower in PGKO males and females. When we profiled serum metabolite concentrations, we found male and female PGKO mice had sexually dimorphic alterations in several metabolites (Table 1). For male PGKO mice, triglyceride and glycerol concentration were increased during ad libitum feeding and short fast, respectively, indicating increased lipolysis. In female PGKO mice, there was a significant increase in non-esterified fatty acid during ad libitum feeding, and lower cholesterol during overnight 


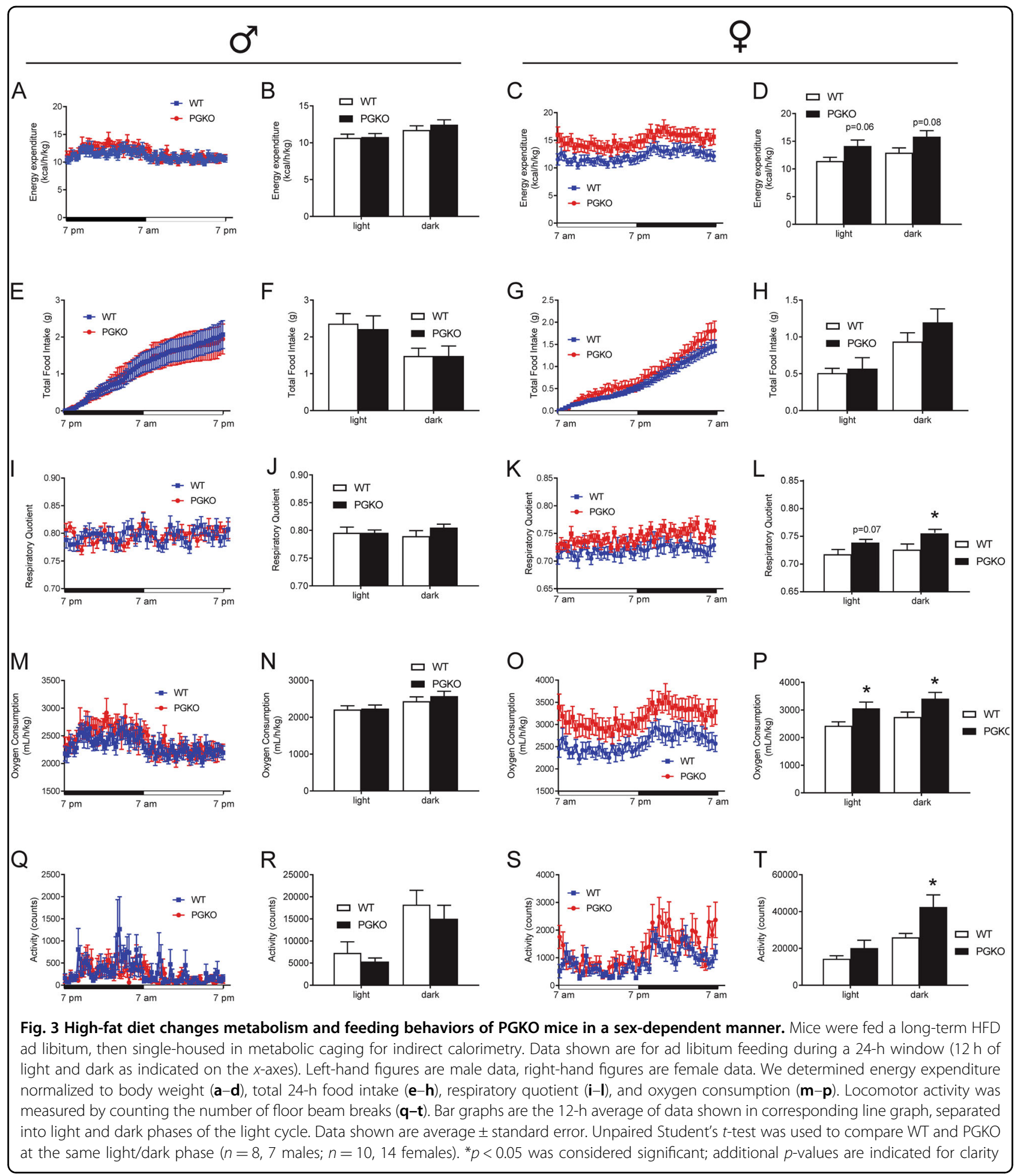

fasting and refeeding. We concluded PGKO mice have increased peripheral metabolism of lipids and that hepatic enzymes were differently regulated to favor lipid utilization in PGKO mice, which could lead to the reduced adiposity gain after prolonged HFD feeding.

\section{PGKO mice have sex-dependent increases in POMC} processing

POMC-derived neuropeptides play critical physiological functions, including appetite regulation ${ }^{27} . \alpha-\mathrm{MSH}$ is anorexigenic, while the effect of $\beta$-endorphin $(\beta-\mathrm{EP})$ on 


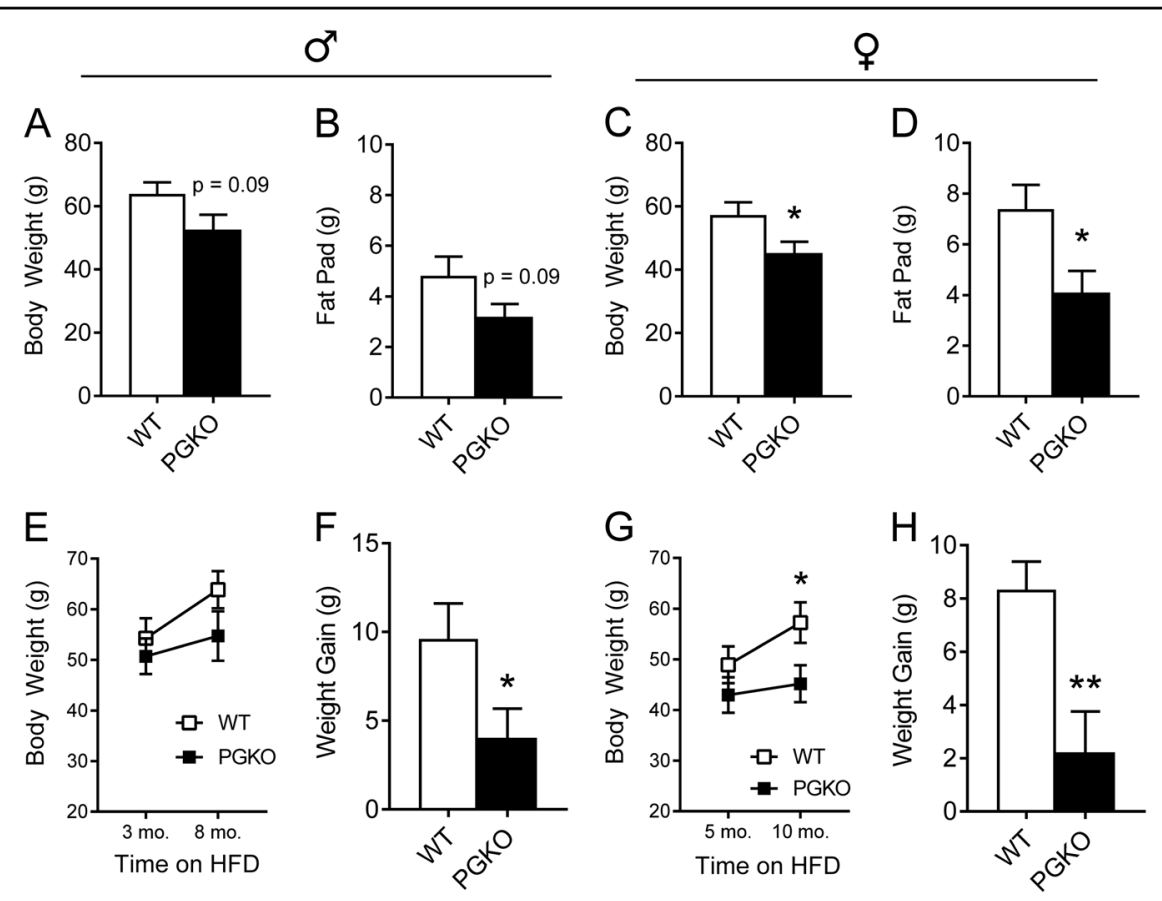

Fig. 4 PGKO mice resisted weight and adiposity gained from long-term HFD. Left-hand figures are male data, right-hand figures are female data. Terminal body weight was measured in male (a) and female (c) mice after 8 and 10 months of HFD, respectively. Fat pad (i.e., epididymal white adipose tissue, EWAT) was dissected from euthanized mice and weighed for males (b) and females (d). Body weight was assessed at the time of indirect calorimetry and 5-months post calorimetry (see Fig. 3) for males (e) and females (g). The difference between these two time points (i.e., weight gain) was calculated for males $(\mathbf{f})$ and females $(\mathbf{h})$. Data shown are average \pm standard error. Statistics were calculated with student's $t$-test ( $n=7,8$ male; $n=9,11$ female). ${ }^{*} p<0.05$ was considered significant; ${ }^{* *} p<0.01$, additional $p$-values are indicated for clarity

satiety is context-dependent ${ }^{26,28,29}$. The bioavailability of $\alpha-\mathrm{MSH}$ and $\beta$-EP is, in part, determined by the expression of $\mathrm{POMC}$ and the subsequent proteolytic processing by $\mathrm{PC} 1, \mathrm{PC} 2$, and $\mathrm{Cpe}$. We measured $\alpha$ $\mathrm{MSH}, \beta-\mathrm{EP}$, and POMC neuropeptides in the mediobasal hypothalamic samples from mice fed HFD for 2 weeks. Prohormone POMC measurement was comparable between sexes and genotypes (Fig. 5a). PGKO mice, especially female knockouts, have increased POMC-derived $\alpha$-MSH and $\beta$-EP (Fig. $5 \mathrm{~b}, \mathrm{c}$ ). This led to greater $\alpha-\mathrm{MSH}: \mathrm{POMC}$ and $\beta$-EP:POMC ratios in PGKO mice (Fig. $5 \mathrm{~d}-\mathrm{e}$ ). Pomc transcript as well as other appetite regulating peptides, Agrp, Pmch, and Npy, were similar in females (Fig. 5f). Since POMC transcripts and peptide were similar or trending downward, we conclude that the observed increases in $\alpha-\mathrm{MSH}$ and $\beta$-EP are not a result of increased POMC synthesis and may result from effects on POMC processing. In addition, there is evidence that endogenous $\beta$-EP may complement the anorexigenic actions of $\alpha-\mathrm{MSH}$ under certain conditions $^{28}$. Taken together, we conclude that higher levels of hypothalamic $\alpha-\mathrm{MSH}$ increases the anorexigenic tone in PGKO mice.

\section{Spontaneous action potential frequency is increased in POMC neurons from PGKO mice}

Since increased electrical activity in POMC neurons suppresses appetite ${ }^{5}$, we used whole cell patch clamp recording in acute brain slices to measure spontaneous activity of POMC neurons in WT and PGKO mice. Gpr17 knockout neurons displayed a higher frequency of spontaneous action potentials (Fig. $5 \mathrm{~g}, \mathrm{j}$ ) and depolarized resting membrane potential $(p=0.08)$ (Fig. $5 \mathrm{~h}, \mathrm{j})$ at basal conditions, but similar AP threshold (Fig. 5i). Then, in order to determine the intrinsic capacity of POMC neurons to be excited by evoked current, we measured the frequency of evoked action potentials (APs) (Fig. 5k), which showed comparable results. We conclude that the increased spontaneous neuronal firing of POMC neurons contributes to the increased anorexigenic tone in PGKO mice.

It has been previously reported that a subset of Agrp/ Npy neurons in the ARH express Pomc during development $^{30}$, which could result in Gpr17 deletion from a subset of Agrp/NPY neurons. To determine the relative number of AgRP/NPY neurons with Gpr17 deletion, we used Npy-Gfp to label AgRP/NPY neurons and 
Reilly et al. Nutrition and Diabetes (2019)9:29

Page 8 of 12

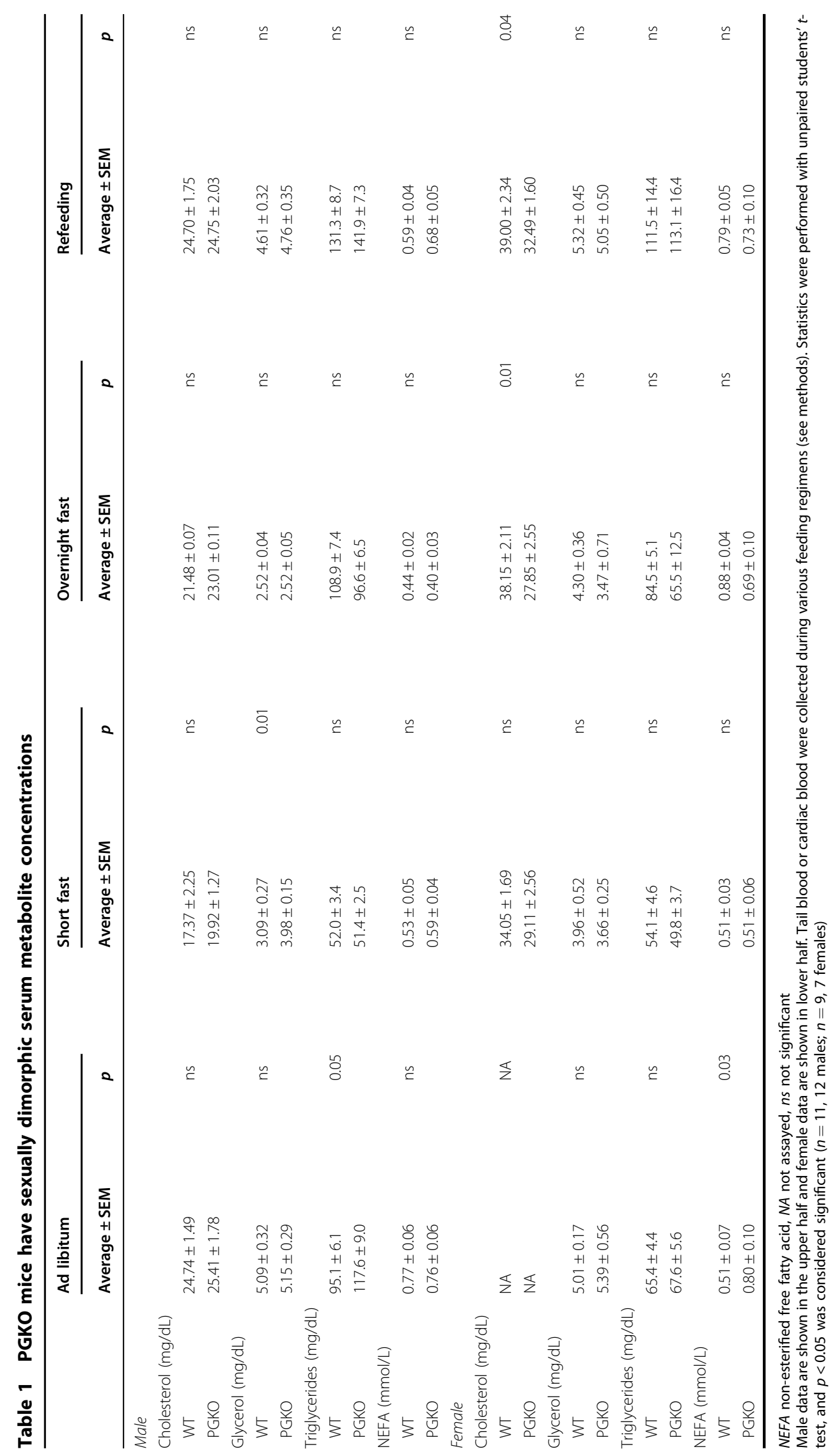

Nutrition and Diabetes 


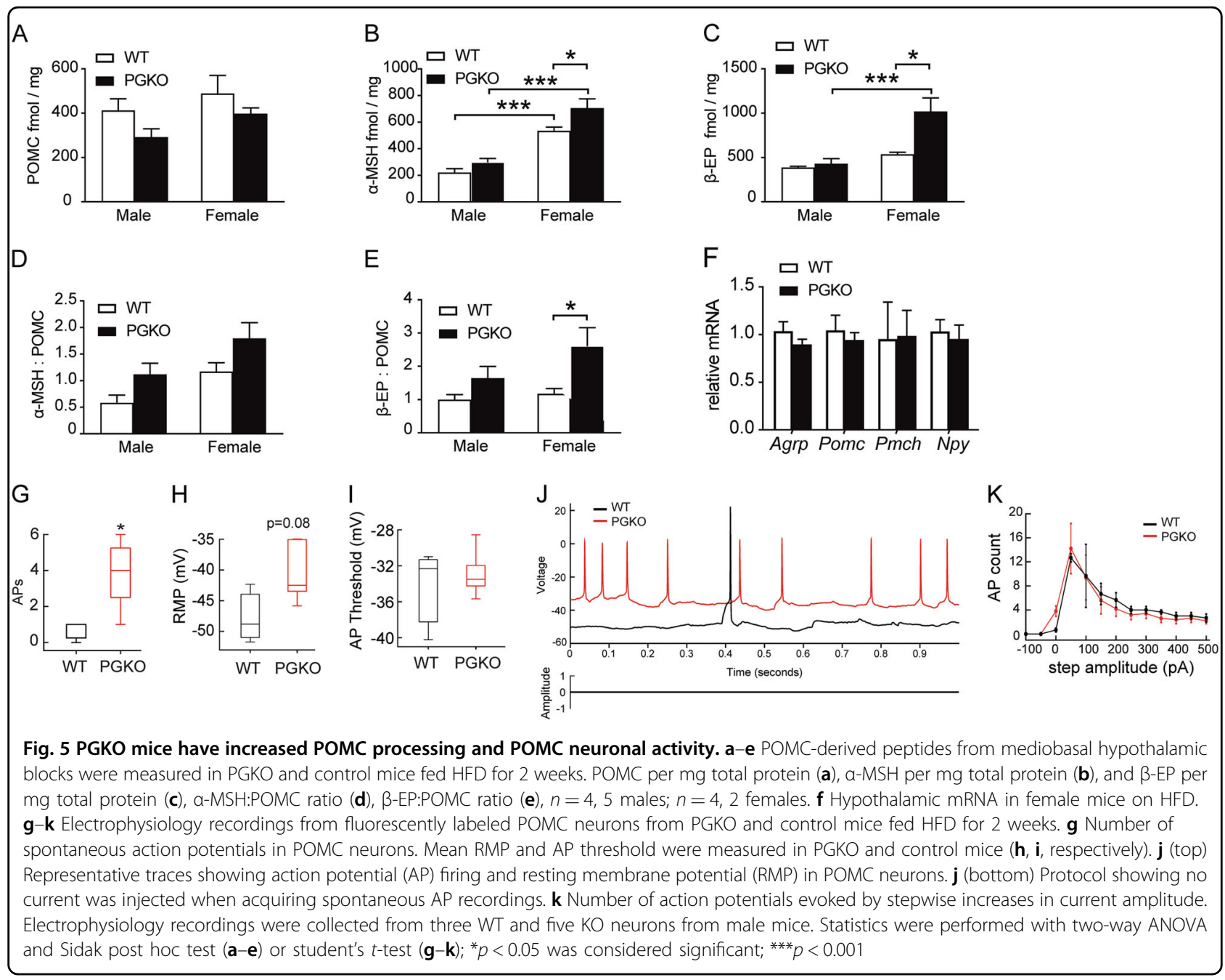

Rosa-tdTomato as a reporter for Pomc-Cre. We were able to identify that $\sim 1 / 3$ of labeled POMC neurons expressed $N p y$-Gfp (Fig. S3A, C). We found that AgRP/NPY neurons in PGKO mice had similar spontaneous action potential (sAP) and resting membrane potential (RMP, Fig. S3D, E). Transcripts encoding orexigenic and anorexigenic neuropeptides had no statistical differences (Fig. 5f). We concluded that Gpr17 deletion in the subset of AgRP/ NPY neurons does not change the orexigenic tone for PGKO mice on NCD.

\section{Leptin signaling in the arcuate cells of PGKO mice}

Leptin increases the excitability of POMC neurons and thus promotes satiety ${ }^{31}$. Therefore, we determined if altered leptin signaling may explain the improved energy balance in Gpr17-deficient mice. In order to determine if PGKO mice had altered sensitivity to leptin in the arcuate nucleus, we used immunohistochemistry to quantify leptin signaling by measuring downstream Stat3 phosphorylation (pStat3). After refeeding, the number of
pStat3 + cells (Fig. S4A, B) and the intracellular pStat3 intensity (Figure S4 C) were modestly increased in PGKO sections, suggesting that PGKO mice have normal sensitivity to leptin on NCD. In HFD-fed mice, the concentration of circulating leptin was unchanged after refeeding (Fig. S4D, E), which suggests that PGKO mice retain normal sensitivity to leptin signaling in the arcuate nucleus.

\section{Discussion}

Our POMC neuron-specific Gpr17 knockout model reveals a physiological role for Gpr17 signaling in regulating energy homeostasis through POMC neurons. PGKO mice were protected from diet-induced obesity, which resulted in lower total body weight and lower EWAT mass for both males and females. Interestingly, female PGKO mice were more protected from long-term obesity than males. We used functional assays of POMC neurons to determine the cellular mechanisms underlying the metabolic phenotypes. We observed 
sex-dependent increases in POMC-derived peptides $(\alpha-\mathrm{MSH}$ and $\beta$-EP) from hypothalamic extracts collected after 2 weeks of HFD exposure in PGKO mice. Our electrophysiological results showed that the spontaneous firing of Gpr17-deficient POMC neurons was increased. We concluded that Gpr17 deletion increased the POMC neuronal activity and promoted better energy homeostasis that curtailed weight gain, especially in female mice. Since male PGKO mice had only partial increases in POMC-derived peptide production compared with female PGKO mice, they were less protected from weight gain.

Sexual dimorphism is well documented for metabolic regulation. Estrogen signaling has been reported as the underlying cause for the gender-specific modulation of abdominal adiposity ${ }^{32}$, adipose tissue lipolysis ${ }^{33}$, and hepatic glucose metabolism ${ }^{34}$. The gender-specific difference in metabolism is more evident with HFD feeding in the adipose and brain tissues ${ }^{34-36}$. Of note, the quantity and physiology of hypothalamic POMC neurons are sex-dependent. In females, POMC neurons are more numerous, have increased electrical activity, and elevated hypothalamic expression of POMC transcript and protein ${ }^{23,37}$. Biological mechanisms regulating POMC neurons are also sex-specific. Leptin signaling in POMC neurons (which increases POMC neuronal firing) also has sex-dependent effects on adipose acquisition, energy expenditure, peripheral glucose tolerance, and locomotor activity ${ }^{38}$. Moreover, estrogen modulates food intake through receptors expressed by POMC neurons ${ }^{39}$. Elucidating how targeting specific signaling pathway in hypothalamic neurons to improve energy homeostasis in a gender-specific manner warrants future investigation.

Hypothalamic neurocircuits, including POMC neurons, have been shown to increase autonomic outflow to peripheral organs, which increases lipolysis in adipose tissues and insulin release by pancreatic islets ${ }^{40-43}$. Our approach for conditional deletion of Gpr17 in POMC neurons resulted in alterations in liver mRNAs related to glucose utilization. This data is consistent with previous findings that connect POMC neuronal activity with hepatic glucose production and insulin sensitivity. For example, chemogenetic stimulation of POMC neurons rapidly induced sympathetic nerve activity to the liver ${ }^{44}$. Central administration of $\alpha-\mathrm{MSH}$ increases hepatic gluconeogenesis and the requisite enzymes Pepck and G6pase $^{45}$. The vagal nerve, which connects brain and liver, suppresses hepatic glucose production ${ }^{46}$. Moreover, insulin action in the CNS alters hepatic glucose production and requires vagal efferents ${ }^{46}$. Therefore, liver mRNAs that are upregulated in PGKO mice could be a direct consequence of altered POMC neuronal activity.
It is also important to note that several age-related mechanisms impair POMC neuronal function and contribute to obesity. Gpr17 knockout may restore some of these pathways that are eroded by aging ${ }^{19}$. For example, as mice age, POMC neurons are electrically silenced by elevated activation of mTOR and hyperactive $\mathrm{K}_{\mathrm{ATP}}$ channels ${ }^{47}$. HFD-fed obese mice have severe leptin resistance in POMC neurons ${ }^{48}$. The synaptic input from AgRP/NPY neurons to POMC neurons increases ${ }^{47,48}$, which progressively increases orexigenic tone in older mice $^{49}$. In our study, PGKO mice, especially females, were protected from adiposity gained due to age and HFD feeding. Although males did not have an apparent change in energy homeostasis, female PGKO mice had increased energy expenditure, nighttime locomotor activity, and increased glucose utilization. It is possible that deficiency in Gpr17 signaling protects against conditions that silence POMC neurons, such as HFD and age.

Our group published previously that Gpr17 substantially altered energy homeostasis in AgRP neuron-specific Gpr17 knockout mice ${ }^{20}$. Since AgRP and POMC neurons have opposing actions on energy homeostasis, one of our principle research questions was whether Gpr17 activity in POMC neurons counteracted that of AgRP neurons. In this regard, PGKO mice surprisingly also had a favorable effect on obesity. One possible explanation is that AgRP conditional knockout mice had enhanced leptin sensitivity of ARH neurons, which was also the trend in PGKO mice. Since leptin signaling oppositely regulates AgRP and POMC neuronal activity, both mice may have favorable metabolic profiles due to sensitization to leptin. The fact that Gpr17 knockout produced positive, but unique, metabolic outcomes in Agrp and Pomc conditional knockout mice supports its potential role as a target for obesity therapy.

In addition, Gpr17 expression is not restricted to POMC and AgRP neurons. Other groups have identified its roles in myelination and metabolic role in oligodendrocytes ${ }^{50}$, mitigating ageing-related defects and ischemic damage in the brain ${ }^{19,51}$ and heart ${ }^{52}$. The inconsistent report on whole-body Gpr17 knockout $^{50,53}$ is probably due to the physiological compensation and developmental adaption that are often associated with germ-line knockouts, which limits the interpretation of negative data as a true absence of a function ${ }^{54}$. Therefore, our studies using conditional knockout mice provide insights on the metabolic function of this orphan GPCR. Future studies with conditional knockout in peripheral tissues are warranted in order to understand the integrative physiology of Gpr17.

In conclusion, our results suggest a role for Gpr17 in regulating energy homeostasis and metabolism through POMC neurons. Thus, eliminating the endogenous agonist or supplying antagonists of Gpr17 could be therapeutically relevant strategies for managing obesity. 


\section{Acknowledgements}

The authors would like to thank Dr. N. Stull for technical help with metabolic caging analysis. The authors also thank members of the Ren lab and the IU Center for Diabetes and Metabolic Diseases for critical discussion of the data. This work was financially supported by National Institute of Diabetes and Digestive and Kidney Diseases R00DK098294; Showalter Scholarship; pilot funding from Indiana Diabetes Center (P30DK097512) and Indiana Clinical and Translational Sciences Institute UL1TR002529 (to H.R.) and the Paul and Carole Stark Fellowship Award (to A.R. and S.Z.) and Indiana University Diabetes and Obesity Research Training Program, T32DK064466 (to A.R.). This work was supported by funding R00DK098294, P\&F grant from P30DK097512 and UL1TR002529, Showalter Scholarship (H.R.), Paul and Carole Stark Fellowship award (A.R. and S.Z.), and Diabetes and Obesity Research Training award T32DK064466 (A.R.).

\section{Author details}

${ }^{1}$ Stark Neurosciences Research Institute, Medical Neuroscience Graduate Program, Indiana University School of Medicine, 320 W. 15th Street Indianapolis, Indianapolis, IN 46202, USA. ${ }^{2}$ Department of Medicine, Division of Endocrinology, Vagelos College of Physicians and Surgeons, Columbia University, New York, NY 10032, USA. ${ }^{3}$ Herman B. Wells Center for Pediatric Research, Department of Pediatrics, Indiana University School of Medicine, 635 Barnhill Drive, Indianapolis, IN 46202, USA. ${ }^{4}$ Center for Diabetes and Metabolic Diseases, Indiana University School of Medicine, 635 Barnhill Drive, Indianapolis, IN 46202, USA. ${ }^{5}$ Department of Pharmacology \& Toxicology, Indiana University School of Medicine, 635 Barnhill Drive, Indianapolis, IN 46202, USA. ${ }^{6}$ Department of Cellular \& Integrative Physiology, Indiana University School of Medicine, 635 Barnhill Drive, Indianapolis, IN 46202, USA. ${ }^{7}$ Department of Biochemistry \& Molecular Biology, Indiana University School of Medicine, 635 Barnhill Drive, Indianapolis, IN 46202, USA

\section{Conflict of interest}

The authors declare that they have no conflict of interest.

\section{Publisher's note}

Springer Nature remains neutral with regard to jurisdictional claims in published maps and institutional affiliations.

Supplementary Information accompanies this paper at (https://doi.org/ 10.1038/541387-019-0096-7).

Received: 25 April 2019 Revised: 12 August 2019 Accepted: 23 August 2019 Published online: 14 October 2019

\section{References}

1. Cone, R. D. The central melanocortin system and energy homeostasis. Trends Endocrinol. Metab. 10, 211-216 (1999).

2. Cone, R. D. Anatomy and regulation of the central melanocortin system. Nat. Neurosci. 8, 571-578 (2005).

3. Mountjoy, K. G., Mortrud, M. T., Low, M. J., Simerly, R. B. \& Cone, R. D. Localization of the melanocortin-4 receptor (MC4-R) in neuroendocrine and autonomic control circuits in the brain. Mol. Endocrinol. https://doi.org/10.1210/ mend.8.10.7854347 (2009).

4. Barsh, G. S. \& Schwartz, M. W. Genetic approaches to studying energy balance: perception and integration. Nat. Rev. Genet. 3, 589-600 (2002).

5. Zhan, C. et al. Acute and long-term suppression of feeding behavior by POMC neurons in the brainstem and hypothalamus, respectively. J. Neurosci. 33, 3624-3632 (2013).

6. Krude, $\mathrm{H}$. et al. Severe early-onset obesity, adrenal insufficiency and red hair pigmentation caused by POMC mutations in humans. Nat. Genet. 19, 155-157 (1998).

7. Morton, G. J., Cummings, D. E., Baskin, D. G., Barsh, G. S. \& Schwartz, M. W. Central nervous system control of food intake and body weight. Nature $\mathbf{4 4 3}$, 289-295 (2006)

8. Cansell, C., Denis, R. G. P., Joly-Amado, A., Castel, J. \& Luquet, S. H. Arcuate AgRP neurons and the regulation of energy balance. Front. Endocrinol. https://www. frontiersin.org/articles/10.3389/fendo.2012.00169/full (2018).
9. Krashes, M. J. et al. Rapid, reversible activation of AgRP neurons drives feeding behavior in mice. J. Clin. Invest. 121, 1424-1428 (2011).

10. Krashes, M. J., Shah, B. P., Koda, S. \& Lowell, B. B. Rapid versus delayed stimulation of feeding by the endogenously released AgRP neuron mediators GABA, NPY, and AgRP. Cell Metab. 18, 588-595 (2013).

11. Aponte, Y., Atasoy, D. \& Sternson, S. M. AGRP neurons are sufficient to orchestrate feeding behavior rapidly and without training. Nat. Neurosci. 14, 351-355 (2011).

12. Cowley, M. A. et al. Integration of NPY, AGRP, and melanocortin signals in the hypothalamic paraventricular nucleus: evidence of a cellular basis for the adipostat. Neuron 24, 155-163 (1999).

13. Spanswick, D., Smith, M. A., Groppi, V. E., Logan, S. D. \& Ashford, M. L. J. Leptin inhibits hypothalamic neurons by activation of ATP-sensitive potassium channels. Nature 390, 521-525 (1997).

14. van den Top, M., Lee, K, Whyment, A. D., Blanks, A. M. \& Spanswick, D. Orexigen-sensitive NPY/AgRP pacemaker neurons in the hypothalamic arcuate nucleus. Nat. Neurosci. 7, 493-494 (2004).

15. Könner, A. C. et al. Insulin action in AgRP-expressing neurons is required for suppression of hepatic glucose production. Cell Metab. 5, 438-449 (2007).

16. Kitamura, T. et al. Forkhead protein FoxO1 mediates Agrp-dependent effects of leptin on food intake. Nat. Med. 12, 534-540 (2006).

17. Plum, L. et al. The obesity susceptibility gene carboxypeptidase $E$ links FoxO1 signaling in hypothalamic pro-opiomelanocortin neurons with regulation of food intake. Nat. Med. 15, 1195-1201 (2009).

18. Ren, H. et al. FoxO1 target Gpr17 activates AgRP neurons to regulate food intake. Cell 149, 1314-1326 (2012).

19. Marschallinger, J. et al. Structural and functional rejuvenation of the aged brain by an approved anti-asthmatic drug. Nat. Commun. 6, 8466 (2015).

20. Ren, H., Cook, J. R., Kon, N. \& Accili, D. Gpr17 in AgRP neurons regulates feeding and sensitivity to insulin and leptin. Diabetes 64, 3670-3679 (2015).

21. Shi, H., Seeley, R. J. \& Clegg, D. J. Sexual differences in the control of energy homeostasis. Front Neuroendocrinol. 30, 396-404 (2009).

22. Shi, H., Sorrell, J. E., Clegg, D. J., Woods, S. C. \& Seeley, R. J. The roles of leptin receptors on POMC neurons in the regulation of sex-specific energy homeostasis. Physiol. Behav. 100, 165-172 (2010).

23. Wang, C. et al. TAp63 contributes to sexual dimorphism in POMC neuron functions and energy homeostasis. Nat. Commun. 9, 1544 (2018).

24. Plum, L. et al. Enhanced PIP 3 signaling in POMC neurons causes $\mathrm{K}_{\text {ATP }}$ channel activation and leads to diet-sensitive obesity. J. Clin. Invest. 116, 1886-1901 (2006).

25. Banks, A. S. et al. SirT1 gain-of-function increases energy efficiency and prevents diabetes in mice. Cell Metab. 8, 333-341 (2008).

26. Panigrahi, S. K. Meece, K. \& Wardlaw, S. L. Effects of naltrexone on energy balance and hypothalamic melanocortin peptides in male mice fed a high-fat diet. J. Endocr. Soc. 3, 590-601 (2019).

27. Cawley, N. X., Li, Z. \& Loh, Y. P. 60 YEARS OF POMC: biosynthesis, trafficking, and secretion of pro-opiomelanocortin-derived peptides. J. Mol. Endocrinol. 56, T77-T97 (2016)

28. Appleyard, S. M. et al. A role for the endogenous opioid $\beta$-endorphin in energy homeostasis. Endocrinology 144, 1753-1760 (2003).

29. Dutia, R., Meece, K. Dighe, S., Kim, A. J. \& Wardlaw, S. L. $\beta$-Endorphin antagonizes the effects of a-MSH on food intake and body weight. Endocrinology 153, 4246-4255 (2012).

30. Padilla, S. L., Carmody, J. S. \& Zeltser, L. M. Pomc-expressing progenitors give rise to antagonistic neuronal populations in hypothalamic feeding circuits. Nat. Med. 16, 403-405 (2010).

31. Cowley, M. A. et al. Leptin activates anorexigenic POMC neurons through a neural network in the arcuate nucleus. Nature 411, 480-484 (2001).

32. Stubbins, R. E., Holcomb, V. B., Hong, J. \& Núñez, N. P. Estrogen modulates abdominal adiposity and protects female mice from obesity and impaired glucose tolerance. Eur. J. Nutr. 51, 861-870 (2012).

33. Benz, V. et al. Sexual dimorphic regulation of body weight dynamics and adipose tissue lipolysis. PLOS One. 7, e37794 (2012).

34. Yan, H. et al. Estrogen improves insulin sensitivity and suppresses gluconeogenesis via the transcription factor Foxo1. Diabetes 68, 291-304 (2019).

35. Grove, K. L., Fried, S. K., Greenberg, A. S., Xiao, X. Q. \& Clegg, D. J. A microarray analysis of sexual dimorphism of adipose tissues in high-fat-diet-induced obese mice. Int J. Obes. (Lond.). 34, 989-1000 (2010).

36. Dorfman, M. D. et al. Sex differences in microglial CX3CR1 signalling determine obesity susceptibility in mice. Nature. Communications 8, 14556 (2017). 
37. Nohara, K. et al. Early-life exposure to testosterone programs the hypothalamic melanocortin system. Endocrinology 152, 1661-1669 (2011).

38. Shi, $\mathrm{H}$. et al. Sexually different actions of leptin in proopiomelanocortin neurons to regulate glucose homeostasis. Am. J. Physiol.-Endocrinol. Metab. 294 E630-E639 (2008).

39. $\mathrm{Xu}, \mathrm{Y}$. et al. Distinct hypothalamic neurons mediate estrogenic effects on energy homeostasis and reproduction. Cell Metab. 14, 453-465 (2011).

40. Madden, C. J. \& Morrison, S. F. Neurons in the paraventricular nucleus of the hypothalamus inhibit sympathetic outflow to brown adipose tissue. Am. J. Physiol.-Regulatory, Integr. Comp. Physiol. 296, R831-R843 (2009).

41. Rosario, W. et al. The brain-to-pancreatic islet neuronal map reveals differential glucose regulation from distinct hypothalamic regions. Diabetes $\mathbf{6 5}$, 2711-2723 (2016).

42. Elmquist, J. K. Hypothalamic pathways underlying the endocrine, autonomic, and behavioral effects of leptin. Int. J. Obes. 25, S78-S82 (2002).

43. Pocai, A. et al. Hypothalamic K(ATP) channels control hepatic glucose production. Nature 434, 1026-1031 (2005).

44. Brandt, C. et al. Food perception primes hepatic ER homeostasis via melanocortin-dependent control of mTOR activation. Cell 175, 1321-1335 (2018).

45. Gutiérrez-Juárez, R., Obici, S. \& Rossetti, L. Melanocortin-independent effects of leptin on hepatic glucose fluxes. J. Biol. Chem. 279, 49704-49715 (2004).
46. Pocai, A., Obici, S., Schwartz, G. J. \& Rossetti, L. A brain-liver circuit regulates glucose homeostasis. Cell Metab. 1, 53-61 (2005).

47. Yang, S.-B. et al. Rapamycin ameliorates age-dependent obesity associated with increased mTOR signaling in hypothalamic POMC neurons. Neuron $\mathbf{7 5}$, 425-436 (2012).

48. Enriori, P. J. et al. Diet-induced obesity causes severe but reversible leptin resistance in arcuate melanocortin neurons. Cell Metab. 5, 181-194 (2007).

49. Newton, A. J. et al. AgRP innervation onto POMC neurons increases with age and is accelerated with chronic high-fat feeding in male mice. Endocrinology 154, 172-183 (2013).

50. Ou, Z. et al. A GPR17-cAMP-lactate signaling axis in oligodendrocytes regulates whole-body metabolism. Cell Rep. 26, 2984-2997 (2019).

51. Bonfanti, E. et al. The role of oligodendrocyte precursor cells expressing the GPR17 receptor in brain remodeling after stroke. Cell Death Dis. 8, e2871 (2017).

52. Cosentino, S. et al. Expression of dual nucleotides/cysteinyl-leukotrienes receptor GPR17 in early trafficking of cardiac stromal cells after myocardial infarction. J. Cell Mol. Med. 18, 1785-1796 (2014).

53. Mastaitis, J. et al. GPR17 gene disruption does not alter food intake or glucose homeostasis in mice. Proc. Natl Acad. Sci. USA 112, 1845-1849 (2015).

54. Chambers, A. P., Sandoval, D. A. \& Seeley, R. J. Integration of satiety signals by the central nervous system. Curr. Biol. 23, R379-R388 (2013). 